Article

\title{
Interspecific Hybridization and Complete Mitochondrial Genome Analysis of Two Ghost Moth Species
}

\author{
Hua Wu ${ }^{1,2}$, Li Cao ${ }^{2}$, Meiyu He ${ }^{2}$, Richou Han ${ }^{2, *}$ and Patrick De Clercq ${ }^{1, *(D)}$ \\ 1 Department of Plants and Crops, Faculty of Bioscience Engineering, Ghent University, Coupure Links 653, \\ 9000 Ghent, Belgium; wuhuaflower@126.com \\ 2 Guangdong Key Laboratory of Animal Conservation and Resource Utilization, Guangdong Public \\ Laboratory of Wild Animal Conservation and Utilization, Institute of Zoology, Guangdong Academy of \\ Science, Guangzhou 510260, China; caol@giabr.gd.cn (L.C.); hemeiyu_xw7@163.com (M.H.) \\ * Correspondence: hanrc@giz.gd.cn (R.H.); patrick.declercq@ugent.be (P.D.C.)
}

Citation: Wu, H.; Cao, L.; He, M.; Han, R.; De Clercq, P. Interspecific Hybridization and Complete Mitochondrial Genome Analysis of Two Ghost Moth Species. Insects 2021, 12, 1046. https://doi.org/10.3390/ insects 12111046

Academic Editors: Robert M. Waterhouse and Marko Mutanen

Received: 8 October 2021

Accepted: 16 November 2021

Published: 21 November 2021

Publisher's Note: MDPI stays neutral with regard to jurisdictional claims in published maps and institutional affiliations.

Copyright: (c) 2021 by the authors. Licensee MDPI, Basel, Switzerland. This article is an open access article distributed under the terms and conditions of the Creative Commons Attribution (CC BY) license (https:/ / creativecommons.org/licenses/by/ $4.0 /)$.
Simple Summary: The Chinese cordyceps is a valuable parasitic Ophiocordyceps sinensis fungusThitarodes/Hepialus larva complex. In view of culturing this complex, a method for the artificial rearing of the Thitarodes/Hepialus ghost moth hosts was established. Deterioration of the host insect population and low mummification rates in infected larvae constrain effective cultivation. Hybridization of Thitarodes/Hepialus populations may overcome this problem. Thitarodes shambalaensis and Thitarodes sp. were inbred or hybridized, and the biological parameters, larval sensitivity to the fungal infection and mitochondrial genomes of the resulting populations were investigated. Hybridization of T. shambalaensis and Thitarodes sp. allowed producing a new generation. One hybrid population (T. shambalaensis females mated with Thitarodes sp. males) showed increased population growth as compared with the parental Thitarodes sp. population. The sensitivity of the inbred larval populations to four fungal isolates of $O$. sinensis differed. The complete mitochondrial genomes of $T$. shambalaensis, Thitarodes sp. and the hybrid population were 15,612 bp, 15,389 bp and 15,496 bp in length, respectively. A + T-rich regions were variable in sizes and repetitive sequences. The hybrid population was located in the same clade with T. shambalaensis, implying the maternal inheritance of mitochondrial DNA.

Abstract: The Chinese cordyceps, a parasitic Ophiocordyceps sinensis fungus-Thitarodes/Hepialus larva complex, is a valuable biological resource endemic to the Tibetan Plateau. Protection of the Plateau environment and huge market demand make it necessary to culture this complex in an artificial system. A method for the large-scale artificial rearing of the Thitarodes/Hepialus insect host has been established. However, the deterioration of the insect rearing population and low mummification of the infected larvae by the fungus constrain effective commercial cultivation. Hybridization of Thitarodes/Hepialus populations may be needed to overcome this problem. The

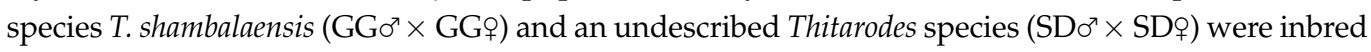
or hybridized to evaluate the biological parameters, larval sensitivity to the fungal infection and mitochondrial genomes of the resulting populations. The two parental Thitarodes species exhibited significant differences in adult fresh weights and body lengths but not in pupal emergence rates. Hybridization of $T$. shambalaensis and Thitarodes sp. allowed producing a new generation. The SD $\sigma^{\top} \times$ GGo population showed a higher population trend index than the SD $\sigma^{7} \times \mathrm{SD}$ \% population, implying increased population growth compared with the male parent. The sensitivity of the inbred larval populations to four fungal isolates of $O$. sinensis also differed. This provides possibilities to create Thitarodes / Hepialus populations with increased growth potential for the improved artificial

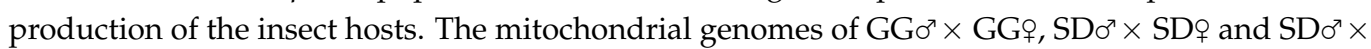
GGo were 15,612 bp, 15,389 bp and 15,496 bp in length, with an A + T content of $80.92 \%, 82.35 \%$ and $80.87 \%$, respectively. The A + T-rich region contains 787 bp with two 114 bp repetitive sequences, $554 \mathrm{bp}$ without repetitive sequences and $673 \mathrm{bp}$ without repetitive sequences in GGo $\times$ GG 9 , SD $0^{\top} \times$ $\mathrm{SD}$ ㅇ and $\mathrm{SD}^{\top} \times \mathrm{GG}$, respectively. The hybrid population $\left(\mathrm{SD}^{\top} \times \mathrm{GG}\right.$ ) $)$ was located in the same clade with GGơ $\times$ GG의, based on the phylogenetic tree constructed by 13 PCGs, implying the maternal inheritance of mitochondrial DNA. 
Keywords: Thitarodes/Hepialus; Ophiocordyceps sinensis; hybridization; infection; mitochondrial genome

\section{Introduction}

The Chinese cordyceps (Ophiocordyceps sinensis fungus-Thitarodes larva complex) is a valued biological resource endemic to the Tibetan Plateau and widely used in medicinal treatments including fatigue, asthma, respiratory and kidney diseases and as health foods, especially in many Asian countries [1-4]. The annual yield of the natural Chinese cordyceps has decreased sharply in recent years due to over-exploitation and habitat degradation $[5,6]$. Due to the extremely limited wild resource and huge market demand, this wild fungusinsect complex costs USD 60,000-75,000 per kilogram [7-11]. Artificial cultivation of the Chinese cordyceps is necessary to protect this valuable bio-resource and to supply commercial trade $[4,11]$.

The insect host species of $O$. sinensis belong to the family Hepialidae (Lepidoptera). Thitarodes armoricanus Oberthür was the first host insect of $O$. sinensis in China to be reported and biologically characterized [12]. Research on the diversity and taxonomy of Hepialidae expanded rapidly during the 1980s. Abundant diversity and phylogeographical structures for the host insects of $O$. sinensis have been reported $[13,14]$. Comparative phylogenetic analyses have suggested coevolutionary relationships between $O$. sinensis and its host insects [14,15]. It appears that most host insect species of the $O$. sinensis fungus have a very narrow distribution on the Tibetan Plateau, and host insect species might vary among different mountain ranges and even from different sides and habitats of the same mountain [16]. Different insect host species or strains show significant differences in morphology, biology and ecology [16-20]. Long-distance dispersal of host insects seems very limited due to the short lifespan of the adult and the heavy abdomens of females that carry eggs; thus most shared genotypes are between strains from adjacent geographic regions [14]. Given the complex and harsh ecological environment in the Tibetan Plateau, most Hepialidae species have a narrow-area distribution type [21]. Of ninety-one named Hepialidae insects spanning thirteen genera reported to be related to host insects of the $O$. sinensis fungus, fifty-seven are considered potential host species of the fungus and are distributed throughout the Tibetan Plateau [17]. However, the described insect host species or strains of $O$. sinensis have never been confirmed by a hybridization technique.

Artificial cultivation of the Chinese cordyceps in low-altitude areas is successful and contains three important milestones: mass production of the host insects, cultivation of effective $O$. sinensis fungus and formation of a fruiting body from the infected insect cadaver. The method for the large-scale artificial rearing of the Thitarodes / Hepialus insect hosts is established $[11,19,20]$. Culture parameters such as food, temperature, humidity and culturing time influence the efficiency of artificial cultivation of different insect species and stages $[19,20]$. The natural foods of Thitarodes/Hepialus larvae are tender roots and buds of plants of the Polygonaceae family such as Polygonum viviparum, P. sphaerostachyum and P. capitatum, as well as other plants in nine different families (e.g., Ranunculaceae, Juncaceae and Cyperaceae) [22]. The roots of Potentilla anserina and carrots (Daucus carota) are favorite larval foods for indoor cultivation [4,23]. Thitarodes species have long and unusual life cycles; it takes 263 to 494 days for Thitarodes jianchuanensis and 443 to 780 days for T. armoricanus to complete the life cycle, including egg, larval instars L1-L9, pupa and adult; the larvae can develop into pupae from the L7, L8 or L9 instar [20]. During the long life cycle and several successive generations in the culture rooms, the insects are usually prone to deterioration $[19,24]$. How to overcome this common phenomenon during insect host rearing is a key issue for the commercial production of the Chinese cordyceps.

Various $O$. sinensis strains from different locations in the Tibetan Plateau have been isolated $[2,14,25]$ and cultured in solid media and liquid media to obtain conidia and blastospores [26]. Stable fruiting body production of $O$. sinensis with mature ascospores 
by artificial media without living insects has also been successfully realized [25]. Two efficient methods are used to infect the host larvae with the $O$. sinensis fungus: the larvae are routinely infected by the mature ascospores collected from the wild Chinese cordyceps or from the artificial fruiting bodies, and the larvae are injected into the hemocoel with the blastospores from the liquid culture containing maltose as a carbon source [26]. In the hemolymph of the larvae, the spindle blastospores exhibit a dimorphic developmental process [26] and may produce exponentially by budding growth and/or grow into elongate hyphal bodies (pseudohyphae) and hyphae by apical growth under the induction of unknown factors [26,27], as reported in the dimorphic fungi Candida albicans and Ustilago maydis [28] and the entomopathogenic fungus Metarhizium rileyi [29]. The living infected host larvae might harbor the spindle blastospores in the hemolymph for several months, contrary to other entomopathogenic fungi such as M. anisopliae and Beauveria bassiana which cause the death of their host larvae within a few days [30,31]. The slow mummification of the larvae post infection is an obstacle for cost-efficient production of Chinese cordyceps $[4,8,11]$. Selection of an $O$. sinensis fungus isolate with high mummifying potential and a Thitarodes insect host species or strain with high sensitivity to the fungal infection should contribute to overcoming this obstacle in the artificial production of the Chinese cordyceps.

Mitochondrial genome sequences have been widely used as molecular markers for diverse evolutionary analyses because of their unique features, including coding content conservation, maternal inheritance and rapid evolution [32]. Insect mitochondrial genomes are usually small closed-circular molecules (15-20 kb) containing 13 proteincoding genes (PCGs), 2 ribosomal RNA (rRNA) genes, 22 transfer RNA (tRNA) genes, and a large non-coding element termed the $\mathrm{A}+\mathrm{T}$-rich or control region [21,33,34]. The mitochondrial gene order also provides important evidence for establishing genome evolutionary relationships $[34,35]$. Due to the improved sequencing technology, insect mitochondrial genomes have been heavily sequenced in recent years. Eight Thitarodes/Hepialus mitochondrial genomes are reported, including Thitarodes renzhiensis (accession number HM744694; size 16,173 bp), Thitarodes yunnanensis (former Ahamus yunnanensis) (accession number HM744695; size 15,816 bp) [21,36], Thitarodes pui (accession numbers KF908880 and MK599283; sizes 15,064 bp and 15,928 bp) [21,37], Hepialus xiaojinensis (accession number KT834973; size 15,397 bp) [38], Hepialus gonggaensis (accession number KP718817; size 15,940 bp) [39], Thitarodes sejilaensis (accession number KU053201; size 15,290 bp;) [40], Thitarodes sp. (accession number KX527574; size 16,280 bp) [41] and Thitarodes damxungensis (accession number MK648145; size 15,362 bp) [21]. With respect to a total of 57 recognizable potential host species of the fungus, the information of the mitochondrial genomes of existing ghost moths is still very limited, and no reports are available on the mitochondrial genomes from the hybrids.

Insight into the biological and molecular characters of the inbred and hybrid populations is elementary for the effective artificial cultivation and evolutionary analysis of these Thitarodes insects. In this study, the hybridization between T. shambalaensis and an undescribed Thitarodes species from two different locations in the Tibetan Plateau was demonstrated. The fitness parameters (such as the number of eggs per female, egg hatching rates, larval fresh weights, larval survival rates, female and male pupal ratios, population trend indexes), larval sensitivity to the fungal infection and mitochondrial genomes of the resulting inbred and hybrid populations were determined to evaluate the hybridization effects.

\section{Materials and Methods}

\subsection{Morphological and Molecular Characteristics of Thitarodes Insect Populations}

The pupae of two Thitarodes insect populations were, respectively, from the mountains in Gongga (referred to as GGo $\times$ GGo $)\left(2476 \mathrm{~m}, 29^{\circ} 70^{\prime} \mathrm{N}, 102^{\circ} 03^{\prime} \mathrm{E}\right.$ ) and Shade (referred to as SD $\left.\circ^{\prime} \times \mathrm{SD}_{+}\right)\left(4560 \mathrm{~m}, 2^{\circ} 65^{\prime} \mathrm{N}, 101^{\circ} 31^{\prime} \mathrm{E}\right)$, Kangding in Sichuan Province, China. 
The valve pattern of the male genitalia is an important characteristic for the morphological identification of Hepialidae insects $[42,43]$. The female and male Thitarodes pupae were differentiated by their genitalia. Briefly, in the last abdominal segment, females exhibit a long longitudinal suture linked to the previous abdominal segment without papillary structures, whereas males exhibit a short longitudinal suture between two papillary structures that is not linked to the previous abdominal segment [44]. The males of GGo $\times$ GGㅇ and $\mathrm{SD}^{\top} \times \mathrm{SD}$ o populations were dissected to show the valve patterns in the laboratory. For the molecular identification of these Thitarodes populations, Cytochrome $b$ and $\operatorname{cox} 1$ sequences were amplified with the primers CB1 (TATGTACTACCATGAGGACAAATATC) and CB2 (ATTACACCTCCTAATTTATTAGGAAT) [42,45] and LCO1490 (GGTCAACAAATCATAAAGATATTGG) and HCO2198 (TAAACTTCAGGGTGACCAAAAAATCA) [46], respectively.

\subsection{Inbred and Hybrid Thitarodes Populations}

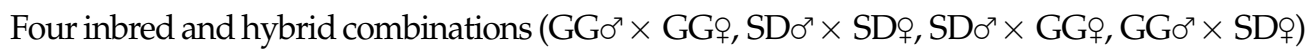
were created with 50 female and 75 male adults for each combination, but the population $\mathrm{GGo}^{2} \times \mathrm{SD}$ \% could not be established due to technical issues related to climatization of the culture room. Three replicates were set up for each combination. The male and female pupae were housed in cartons $(\mathrm{L}=104 \mathrm{~cm} ; \mathrm{W}=50 \mathrm{~cm} ; \mathrm{H}=50 \mathrm{~cm})$ with moist moss at $9-17^{\circ} \mathrm{C}$ and $50-80 \%$ relative humidity. When the adults emerged, they were housed in small cylindric nets $(\mathrm{D}=28 \mathrm{~cm}$; $\mathrm{H}=32 \mathrm{~cm}$ ) to allow mating for 3-5 days. The collected eggs from the mated females were transferred to a culture room and maintained at $9-13{ }^{\circ} \mathrm{C}$ to establish the experimental populations in the Institute of Zoology, Dongguan (43 m above sea level), Guangdong Province, China.

To evaluate the development, survival, fertility and sensitivity to the fungal infection in the resulting Thitarodes populations, $600 \mathrm{eggs}$ from each inbred or hybrid combination were surface-sterilized for $3 \mathrm{~min}$ with a solution containing $2.5 \mathrm{~mL}$ of $4 \mathrm{M} \mathrm{NaOH}, 0.5 \mathrm{~mL}$ of $12 \% \mathrm{NaOCl}$ and $21.5 \mathrm{~mL}$ of distilled water [47], rinsed 3 times with sterile distilled water and placed in a sterile plastic container $(\mathrm{L}=48 \mathrm{~cm} ; \mathrm{W}=35 \mathrm{~cm} ; \mathrm{H}=17 \mathrm{~cm})$ containing $2 \mathrm{~kg}$ coconut peat $\left(65 \%\right.$ of water content) and $1 \mathrm{~kg}$ Potentilla anserina roots as food at $9-13{ }^{\circ} \mathrm{C}$. For each hybridization combination, 30 containers were established. When the larvae reached the third instar, they were individualized into a plastic cup $(\mathrm{D}=3.5 \mathrm{~cm} ; \mathrm{H}=6.5 \mathrm{~cm})$ with the same peat and food as above (15 $\mathrm{g}$ coconut peat and $15 \mathrm{~g}$ food for each cup) to avoid larval cannibalism [20]. Fresh food was added to each cup every 2 months to obtain 6 th instar larvae (average fresh weight $=0.52 \pm 0.03 \mathrm{~g}$ ) for fungal infection by the injection method. The larval number in each container was recorded, and the average hatch rate was calculated. At the sample date (every 30 days), the survival rates, longevity, fresh weight, body length and sex proportion of pupae and adults and fecundity were recorded.

\subsection{O. sinensis Fungal Isolates}

$\mathrm{KD}, \mathrm{YN}, \mathrm{XZ}$ and $\mathrm{QH}$ fungal isolates of $\mathrm{O}$. sinensis isolated from the fruiting bodies of wild Chinese cordyceps, respectively, from Sichuan, Yunnan, Tibet and Qinghai, China, were cultured on PPDA medium (liquid PPDA medium: 200 g potato extract, $20 \mathrm{~g}$ glucose, $10 \mathrm{~g}$ peptone, $1.5 \mathrm{~g} \mathrm{KH}_{2} \mathrm{PO}_{4}, 0.5 \mathrm{~g} \mathrm{MgSO}_{4}, 20 \mathrm{mg}$ vitamin $\mathrm{B}_{1}$ and $1000 \mathrm{~mL}$ distilled water; solid PPDA medium: $15 \%$ agar in liquid PPDA medium) at $13{ }^{\circ} \mathrm{C}$. The fungal isolates were identified by using the amplified sequence from the internal transcribed spacer (ITS; ITS15.8S-ITS2) of the nuclear ribosomal DNA as described by [48]. The identified O. sinensis isolates were preserved at $-80{ }^{\circ} \mathrm{C}$ in the Institute of Zoology, Guangdong Academy of Science, Guangzhou, China.

The fungal colonies cultured on the PPDA plates at $13{ }^{\circ} \mathrm{C}$ for 60 days were transferred to $250 \mathrm{~mL}$ flasks containing $150 \mathrm{~mL}$ liquid PM medium (200 g potato extract, $20 \mathrm{~g}$ maltose, $10 \mathrm{~g}$ peptone, $1.5 \mathrm{~g} \mathrm{KH}_{2} \mathrm{PO}_{4}, 0.5 \mathrm{~g} \mathrm{MgSO}_{4}, 20 \mathrm{mg}$ vitamin $\mathrm{B}_{1}$ and $1000 \mathrm{~mL}$ distilled water) [26]. The flasks were incubated on a $120 \mathrm{rpm}$ shaker at $13{ }^{\circ} \mathrm{C}$, the blastospores from the flasks were harvested after 50 days by using three layers of sterile lens papers to remove hyphae and large particles, and the filtered solution was centrifuged at $8000 \mathrm{rpm}$ for $15 \mathrm{~min}$ at $10{ }^{\circ} \mathrm{C}$. The harvested blastospores were re-suspended in sterile phosphate-buffered 
saline (PBS; pH 7.0) at a concentration of $3.0 \times 10^{6}$ blastospores per $\mathrm{mL}$ and kept at $4{ }^{\circ} \mathrm{C}$ for less than 3 days before use for larval infection.

\subsection{Larval Infection of Inbred Populations by O. sinensis Isolates}

The larvae from 2 inbred populations $\left(\mathrm{GG} 0^{\top} \times \mathrm{GG}\right.$, $\mathrm{SD} 0^{\top} \times \mathrm{SD}$ ) were injected with $\mathrm{KD}, \mathrm{YN}, \mathrm{XZ}$ or $\mathrm{QH}$ fungal isolates of $O$. sinensis. An aliquot of $4 \mu \mathrm{L}$ blastospore suspension containing $1.2 \times 10^{4}$ blastospores was injected into each 6 th instar larva by a microinjection system (IM-31; Narishige, Tokyo, Japan). One hundred and eighty larvae were used for each replicate, and three replicates were set for each injection. Larvae injected with PBS buffer or without any injection were set as controls. The injected larvae were reared at $4{ }^{\circ} \mathrm{C}$ for one week and then transferred to a culture room at $13^{\circ} \mathrm{C}$. After 90 days, about $10 \mu \mathrm{L}$ of hemolymph of each injected larva (6th instar) was sampled to confirm the presence of the growing blastospores stained by Calcofluor White (Sigma, Kanagawa, Japan) and observed by a fluorescence microscope (IX73; Olympus, Tokyo, Japan). The injected larvae were reared at $13{ }^{\circ} \mathrm{C}$ until the larvae became stiff and were coated with growing mycelia. The mummified larvae with head upward were then planted into soil of $55-60 \%$ humidity to induce the formation of stroma at $4{ }^{\circ} \mathrm{C}$. The survival and mummification of the injected larvae were monthly checked. Data on larval infection of the hybrid populations could not be gathered due to an insufficient number of larvae available for fungal injection.

\subsection{Analysis of the Mitochondrial Genomes}

Three male adults from $\mathrm{GGo}^{\top}$ and $\mathrm{SD} 0^{\top}$ and three larvae from SDo ${ }^{\top} \times \mathrm{GG}$ i in dry ice were used for mitochondrial genome sequencing by Shanghai BIOZERON Co., Ltd., with the routine method [41]. The nucleotide sequences of protein-coding genes (PCGs) from the annotated mitochondrial genomes were translated to protein sequences using the invertebrate mitochondrial code. For the base composition of the nucleotide sequences, the composition skewness was calculated as follows: AT skew $=[\mathrm{A}-\mathrm{T}] /[\mathrm{A}+\mathrm{T}], \mathrm{GC}$ skew $=[G-C] /[G+C][49]$. Thirteen PCGs and two rRNA genes were inferred based on comparison with mitochondrial genomes of 10 previously sequenced Hepialidae species (T. damxungensis, T. gonggaensis, T. pui, T. renzhiensis, T. sejilaensis, Thitarodes sp., H. xiaojinensis, T. yunnanensis, Napialus hunanensis, Endoclita signifer). The location and secondary structures of the 22 tRNA were predicted by tRNAscan-SE (http:/ /owelab.ucsc.edu/ tRNAscan-SE/) (accessed on 13 November 2020). After the removal of the termination codon, the codon usage frequency and the first, second and third base use frequency of the codon were calculated using MEGA 7.0. The overlapping regions and intergenic spacers between genes were manually counted. The entire A + T-rich region was subjected to a search for the tandem repeats using the Tandem Repeats Finder program [50].

To construct the phylogenetic relationships within Hepialidae in Lepidoptera, 10 complete mitochondrial genomes of the above hepialid species were downloaded from GenBank. Drosophila melanogaster was used as an outgroup. A maximum likelihood (ML) tree was built in MEGA 7.0 using the nucleotide sequence of 13 PCGs, based on the "find best DNA/protein models (ML)". The "GTR + G" model was chosen for phylogenetic analysis because it produced the lowest values for both the BIC (Bayesian information criterion) and the AICc (corrected Akaike information criterion). The confidence values of the ML tree were evaluated via a bootstrap test with 1000 iterations.

\subsection{Data Analysis}

The data are expressed as means \pm SE. The average survival rates, fresh weight, body length and sex proportions of pupae and adults at the sample time points were determined. In addition, a population trend index (I) was calculated with $\mathrm{I}=\mathrm{P}_{\mathrm{II}} / \mathrm{P}_{\mathrm{I}}$, where $\mathrm{P}_{\mathrm{I}}=$ numbers of pupae in the parental generation, and $\mathrm{P}_{\mathrm{II}}=$ numbers of pupae in the next generation [20]. The data were analyzed with SPSS 21.0 (SPSS Inc., Chicago, IL, USA) to compare the differences among the treatments. Differences among means by Tukey's multiple-range test were considered significant at $p<0.05$. 


\section{Results}

\subsection{Morphological and Molecular Identification of Two Thitarodes Species}

The SDo $\times$ SDo population from Sichuan Province, China, was considered to be an undescribed Thitarodes species, by the phylogenetic analysis, although the valve pattern of the male genitalia of $\mathrm{SD}^{-1} \times \mathrm{SD}$ r resembled that of Thitarodes kangdingensis (Figure 1) (Prof. Zhiwen Zou, personal communication). The GGo $\times$ GGo population also from Sichuan Province, China, was confirmed to be Thitarodes shambalaensis [43], based on the valve pattern of the male genitalia (Figure 1) and the sequences of the cox 1 fragment. The complete cox 1 sequences of the two species were $1531 \mathrm{bp}$ and were submitted to GenBank (accession numbers OK104111 and OK047724, respectively).

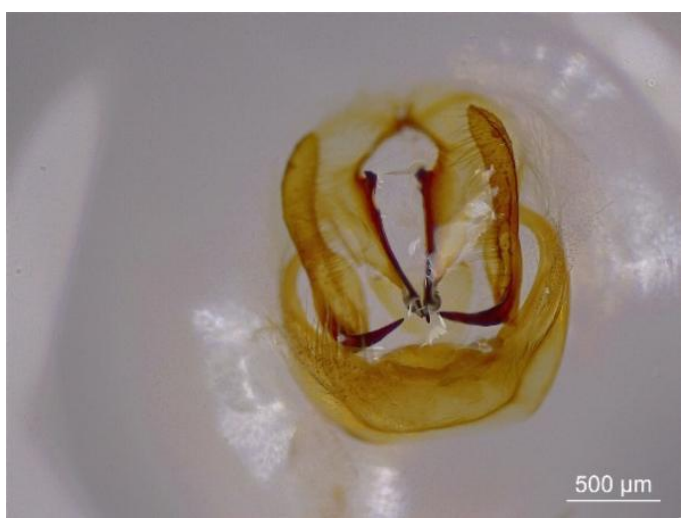

(a)

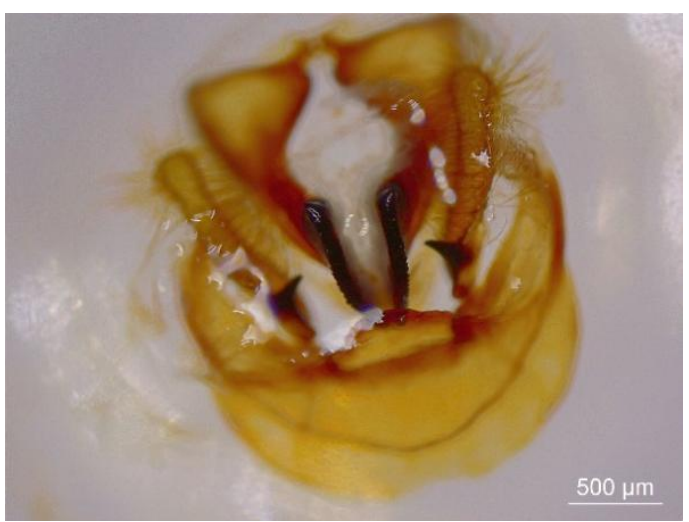

(b)

Figure 1. The valve patterns in male genitalia of Thitarodes $\mathrm{sp} .\left(\mathrm{SD}^{\top} \times \mathrm{SD}\right.$ ) $)(\mathbf{a})$ and Thitarodes shambalaensis $\left(\mathrm{GG} 0^{\top} \times \mathrm{GG}\right.$ ) (b). Bars $=500 \mu \mathrm{m}$.

\subsection{Development from the Pupae to Next-Generation Pupae in Inbred and Hybrid Populations}

The data on the fresh weight and body length of the pupae from the parental species were collected. The fresh weights were $0.88 \pm 0.04 \mathrm{~g}$ and $0.67 \pm 0.03 \mathrm{~g}$ for SDo $\times$ SDo and GGo $\times$ GGo female pupae and $0.59 \pm 0.05 \mathrm{~g}$ and $0.45 \pm 0.03 \mathrm{~g}$ for SDo $\sigma^{7} \times \mathrm{SD}$ \% and GGo $\times$ GGo male pupae, respectively. The body lengths were $2.73 \pm 0.21 \mathrm{~cm}$ and $2.50 \pm 0.05 \mathrm{~cm}$

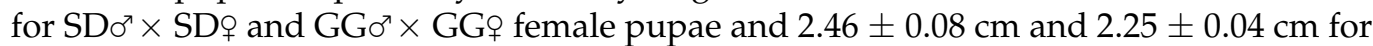
$\mathrm{SD}^{\top} \times \mathrm{SD}$ ㅇ and GG $\circ^{\top} \times$ GGㅇ male pupae, respectively (Table 1). The pupae developed into adults in 30-45 days at $9-17^{\circ} \mathrm{C}$. No significant differences were found for the fresh weights and body lengths of the pupae between the two populations, except for the fresh weight of female pupae, which differed significantly between the populations (Table 1). The ratios of females and males in the pupae (SD $\sigma^{\top} \times \mathrm{SD}$ : $1.27 \pm 0.16$; GGo $\times$ GGo: $1.04 \pm 0.06$ ) and adults (SDo $\times$ SDo: $0.89 \pm 0.06$; GGo $\times$ GGo: $0.87 \pm 0.03)$ were also not significant. The emergence rates were $38.38 \%$ and $48.96 \%$ for $\mathrm{SD}^{\top} \times \mathrm{SD}$ 웅 and GGo $\times$ GGo females and $43.57 \%$ and $57.58 \%$ for $\mathrm{SD}^{\top} \times \mathrm{SD}$ \% and GGo $\times$ GG \% males, respectively, showing no significant differences between the two parental insect populations (Table 1).

The females and males of the GGor $\times$ GGo population were mating all day and night,

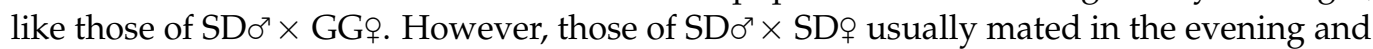
at night. The adults did not feed, and their life span usually lasted 5-7 days at 9-17 ${ }^{\circ} \mathrm{C}$. Contrary to the clean eggs from GGo $\circ^{\top} \times \mathrm{GG}^{\circ}$, the eggs from $\mathrm{SD}{ }^{\top} \times \mathrm{SD}$ \% were coated with a sticky secretion. The average number of eggs per female for SD $\sigma^{\top} \times \mathrm{SD}$ (512 \pm 3 ) was significantly higher than that for the other populations, whereas the number of eggs in one milliliter (3976 \pm 109 ) was higher and the weight of one thousand eggs $(0.18 \pm 0.01 \mathrm{~g})$ was lower for $\mathrm{SD}^{\top} \times \mathrm{SD}$ \% compared with other populations, indicating the smaller egg size of $\mathrm{SD}^{\top} \times \mathrm{SD}$ ㅇ $\left(\right.$ Table S1). The hatching rate for SD $\circ^{7} \times \mathrm{GG}$ 우 $(12.62 \pm 2.80 \%)$ was significantly lower than that for the other populations (Table S1). Thus, these two insect populations exhibited characteristic differences in mating behavior, egg size and the presence of a sticky 
secretion coating the eggs but showed no significant differences in the ratio of female and male pupae or in the pupal emergence rates.

Table 1. Morphological and biological characters of Thitarodes sp. and T. shambalaensis.

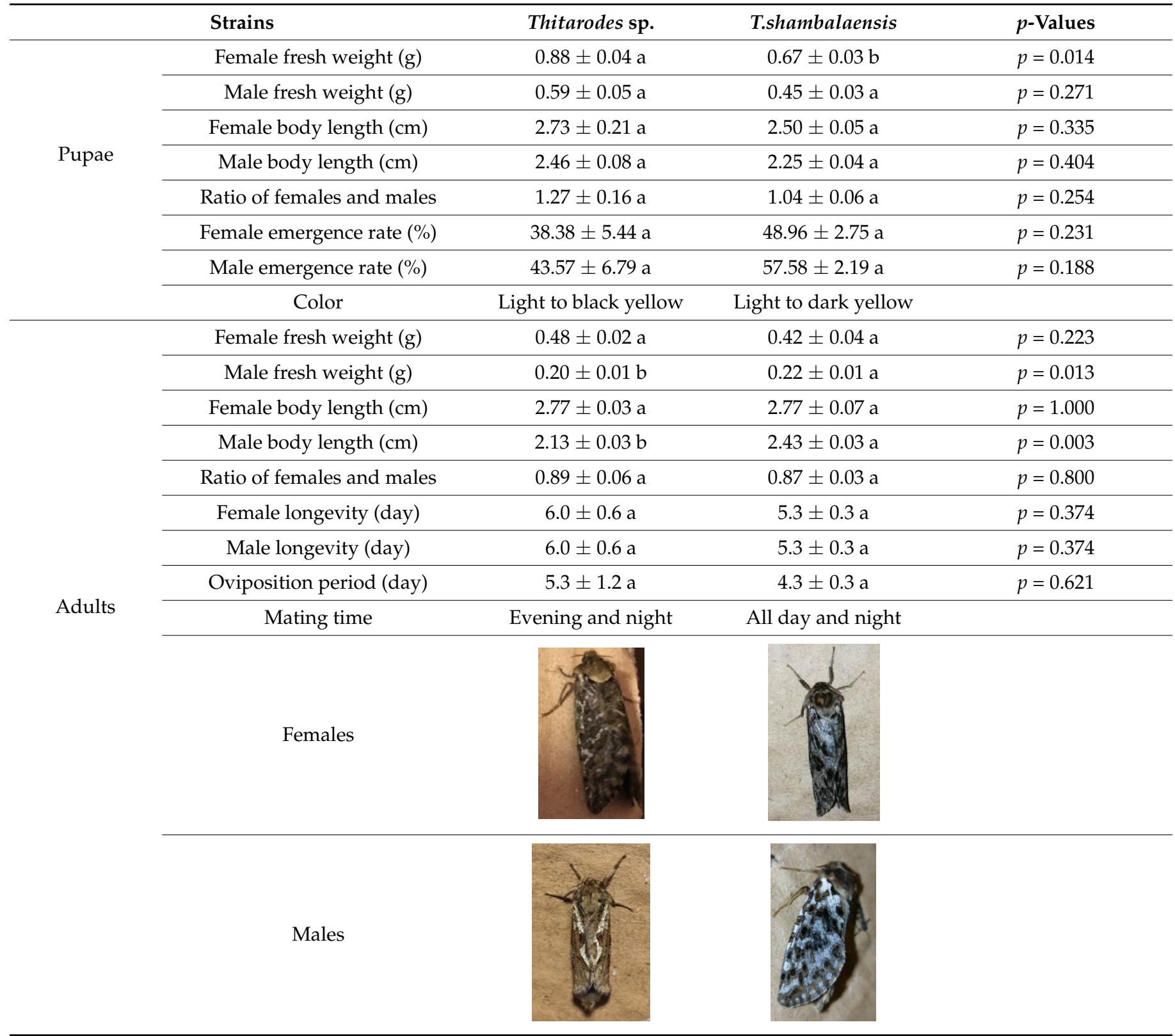

Note: the rows with the same letters indicated no significant differences $(p>0.05)$.

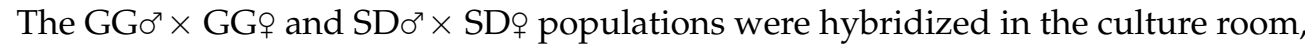
and the larvae successfully became pupae in the resulting hybrid populations. The fresh weights of the larvae from the inbred and hybrid populations did not vary significantly with culture times in 12 months (Figure S1). The survival rates of the larvae were stable at $80-100 \%$ in the first 10 months but decreased sharply after 11 months, especially in the $\mathrm{SD}^{\top} \times \mathrm{SD}$ ? and SD $\circ^{\top} \times \mathrm{GG}$ q populations (Figure S2), due to the increasing larval mortality before pupation. The larvae became pupae after 22 months. The ratios of the resulting female and male pupae were $0.28 \pm 0.15$ for $\mathrm{SD}^{\top} \times \mathrm{SD}$,, $0.61 \pm 0.05$ for $\mathrm{SD} \odot^{\top} \times \mathrm{GG}$ q and $0.78 \pm 0.03$ for GGo $\times$ GGㅇ, which were significantly different. Population trend index values were quite variable, with $\mathrm{I}=0.01$ for $\mathrm{SD}^{7} \times \mathrm{SD}$ 우 0.32 for $\mathrm{SD} \sigma^{7} \times \mathrm{GG}$ 우 2.25 for $\mathrm{GG}^{7} \times \mathrm{GG}$, indicating different proportions of pupal numbers in the previous generation 
over the next generation in the inbred and hybrid populations. The adults emerged from the pupae after 23 months. Unfortunately, the experiments were discontinued due to the emergence of too few adults caused by the high mortality $(>70 \%)$ of the pupae. Nonetheless, the above results indicate that hybridization of Thitarodes sp. and T. shambalaensis allowed harvesting a next generation of adults at least from the SD $\sigma^{7} \times \mathrm{GG}$ q population.

\subsection{Larval Infection of Inbred Populations by O. sinensis Isolates}

As shown in Table S2, the percentages of the larvae carrying the blastospores varied at 120 days post infection, from $20.37 \pm 5.38 \%$ for the larvae of GGo $\times$ GGo injected with fungal isolate $X Z$ to $62.96 \pm 1.96 \%$ for the larvae of GGo' $\times$ GGo injected with fungal isolate $\mathrm{QH}$; for $\mathrm{SD}^{\top} \times \mathrm{SD}$, the mummification rate of the larvae containing fungal isolate $\mathrm{XZ}$ was significantly lower than that of those containing fungal isolates $\mathrm{KD}, \mathrm{QH}$ and $\mathrm{YN}$; for GGo $\times$ GG, the mummification rate of the larvae containing fungal isolate $X Z$ was significantly lower than that of those containing fungal isolates $\mathrm{KD}, \mathrm{QH}$ and $\mathrm{YN}$. For fungal isolates KD, $\mathrm{XZ}$ and $\mathrm{YN}$, no significant differences in mummification rate were observed among the two infected larval populations (Table S2). Sixty and 90 days after infection, no significant differences in the percentages of the larvae carrying blastospores were found among both larval populations. Although the larval hemocoel was filled with growing blastospores after 90 days, the fresh weights of the larvae in both populations did not differ markedly (Table S3).

\subsection{Mitochondrial Genome Analysis}

Organization and base composition. The complete mitochondrial genomes of two

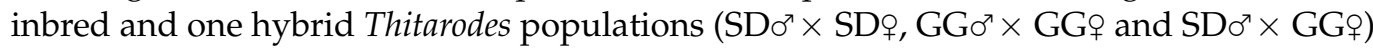
were a circular DNA molecule of 15,389 bp, 15,612 bp and 15,496 bp in length, respectively (accession number: MZ675586, MZ675587 and MZ675588) (Figure 2). Like most other metazoan mitochondrial genomes, each of three Thitarodes mitochondrial genomes contained 13 PCGs, 22 tRNAs, 2 rRNAs and a large non-coding control region. Among the 37 genes in each mitogenome, there were 9 PCGs and $14 \mathrm{tRNAs}$ encoded in the heavy strand, while 4 PCGs, 8 tRNAs and 2 rRNAs were encoded in the light strand. The mitochondrial genome structure was compact. The gene order of the Thitarodes mitochondrial genomes was uniform (Figure 2).

The mitochondrial genome content of these three Thitarodes populations was A + T-biased, ranging from $80.87 \%\left(\mathrm{SD}^{\top} \times \mathrm{GG}\right.$ ) $)$ to $82.35 \%\left(\mathrm{SD}^{\top} \times \mathrm{SD}\right.$ ) (Table 2$)$. The $\mathrm{A}+\mathrm{T}$ content of the $\mathrm{SD}^{7} \times \mathrm{SD}$ m mitochondrial genome was $82.35 \%$, which was larger than that of the other two mitochondrial genomes. The AT skew in the forward strand of the SD ${ }^{\top} \times \mathrm{SD}$ m mitochondrial genome was slightly positive (0.008), which was different from the other two mitochondrial genomes (0.020) (Table 2). Likewise, The GC skew of the SD $\sigma^{\top} \times$ SD o mitogenome $(-0.182)$ was also obviously different from those of the other two $(-0.231$ to -0.234$)$.

Protein-coding genes. The 13 PCGs in these mitochondrial genomes included 7 NADH dehydrogenase subunits (nad1-6, nad4L), 3 cytochrome c oxidase subunits (cox1-3), 2 ATPase subunits $(a t p 6, a t p 8)$ and one cytochrome b gene $(c y t b)$. The lengths of the 13 PCGs in the mitochondrial genomes of $\mathrm{SD}^{\top} \times \mathrm{SD}$ ㅇ, GGo ${ }^{\top} \times \mathrm{GG}$ ㅇ and SD $\varsigma^{\top} \times \mathrm{GG}$ ㅇ were 11,073, 11,067 and 11,067, respectively (Table 2). When the termination codons were excluded, the

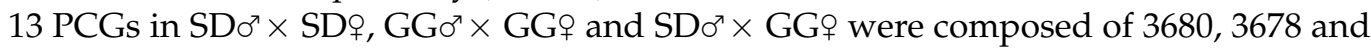
3678 codons, respectively. These findings indicate a high degree of similarity in the PCG code number among the three mitochondrial genomes (Figure 3). 

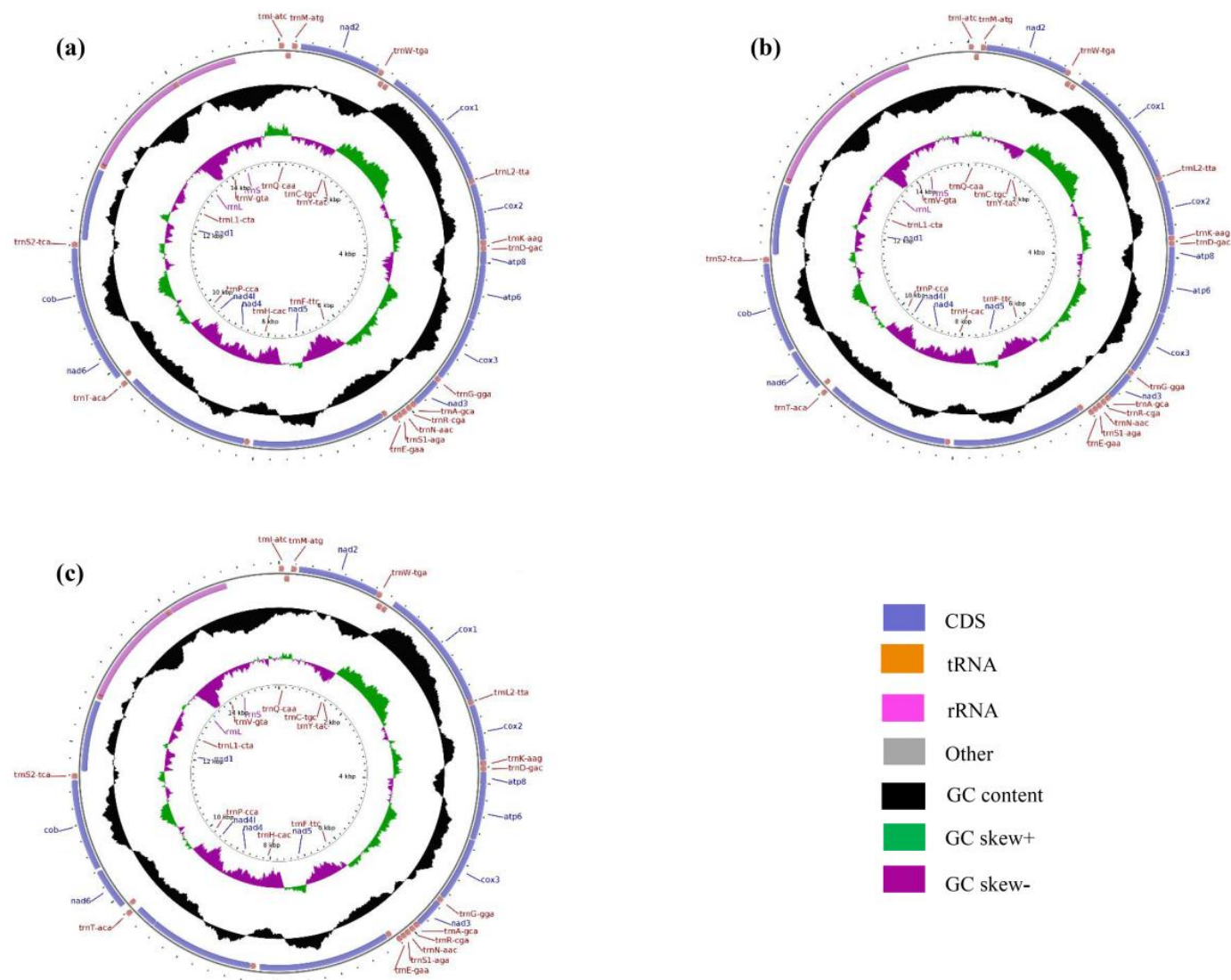

Figure 2. Circular map of the mitochondrial genomes of Thitarodes populations: $\mathrm{SD} 0^{\top} \times \mathrm{SD}$ 우 (a), GGơ $\times \mathrm{GG}$ ㅇ $(\mathbf{b})$ and $\mathrm{SD}^{\top} \times \mathrm{GG}$ ( $)$.
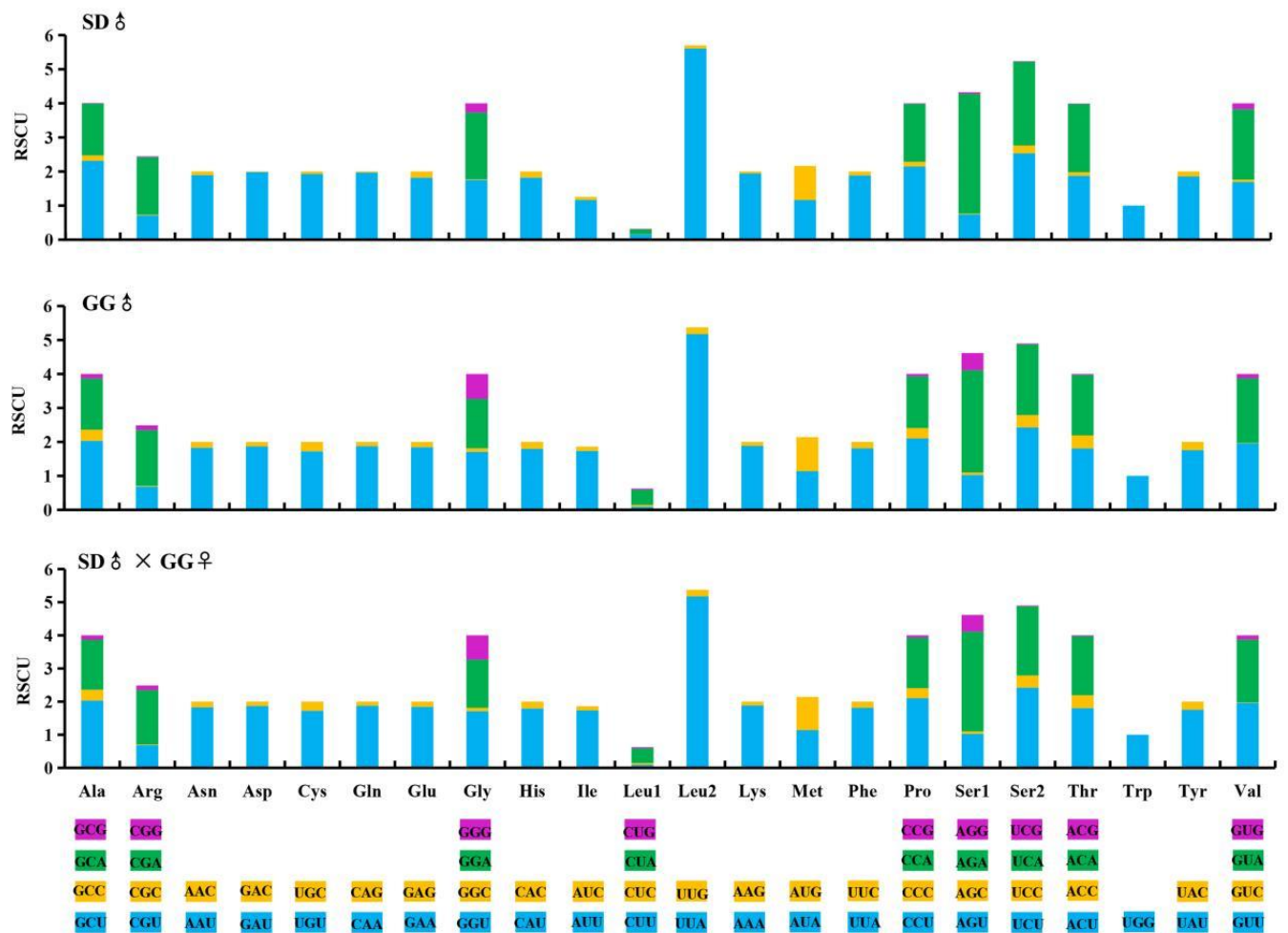

Figure 3. Codon usage numbers (N) and the relative synonymous codon usage (RSCU) among three Thitarodes mitochondrial genomes. 
Table 2. Comparison of the complete mitochondrial genomes of three Thitarodes populations.

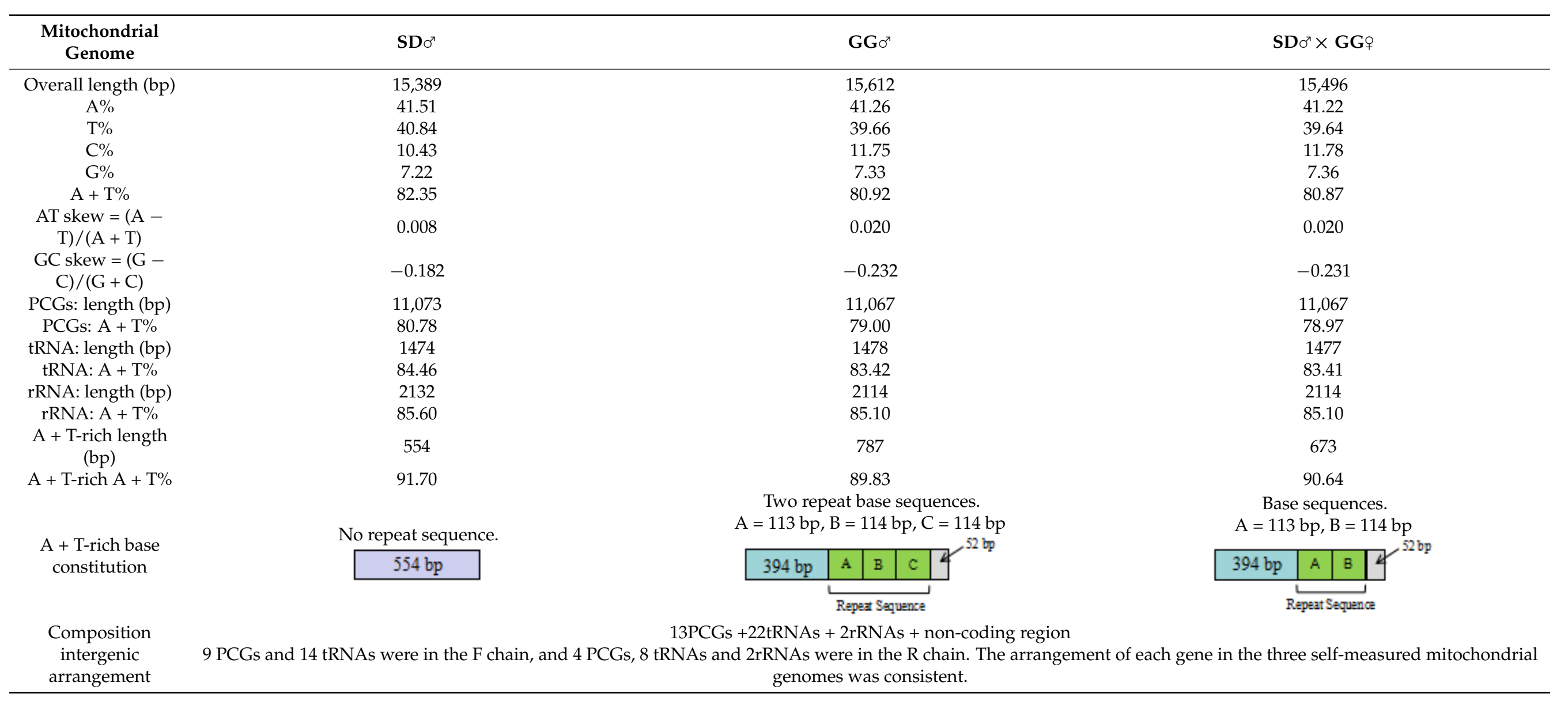


Table 2. Cont.

\begin{tabular}{|c|c|c|c|c|c|c|c|c|c|c|c|c|c|c|c|c|}
\hline Gene & Chain & Location & Spacer & Length & $\begin{array}{c}\text { Initiation } \\
\text { code }\end{array}$ & $\begin{array}{l}\text { Termination } \\
\text { code }\end{array}$ & Location & Spacer & Length & $\begin{array}{l}\text { Initiation } \\
\text { code }\end{array}$ & $\begin{array}{l}\text { Termination } \\
\text { code }\end{array}$ & Location & Spacer & Length & $\begin{array}{l}\text { Initiation } \\
\text { code }\end{array}$ & $\begin{array}{l}\text { Termination } \\
\text { code }\end{array}$ \\
\hline trnI & F & $1-67$ & - & 67 & & & $1-65$ & - & 65 & & & $1-64$ & - & 64 & & \\
\hline $\operatorname{trn} Q$ & $\mathrm{R}$ & $78-146$ & 10 & 69 & & & 63-131 & -3 & 69 & & & $62-130$ & -3 & 69 & & \\
\hline $\operatorname{trn} \widetilde{M}$ & F & $158-227$ & 11 & 70 & & & $151-220$ & 19 & 70 & & & $148-217$ & 17 & 70 & & \\
\hline $\operatorname{trn} W$ & F & 1249-1314 & 1 & 66 & & & 1239-1304 & -2 & 66 & & & $1236-1301$ & -2 & 66 & & \\
\hline $\operatorname{trn} C$ & $\mathrm{R}$ & $1307-1373$ & -8 & 67 & & & $1297-1367$ & -8 & 71 & & & $1294-1364$ & -8 & 71 & & \\
\hline $\operatorname{trn} Y$ & $\mathrm{R}$ & $1379-1444$ & 5 & 66 & & & $1374-1440$ & 6 & 67 & & & $1371-1437$ & 6 & 67 & & \\
\hline $\operatorname{cox} 1$ & F & $1447-2977$ & 2 & 1531 & CGA & $\mathrm{T}$ & $1443-2973$ & 2 & 1531 & CGA & $\mathrm{T}$ & $1440-2970$ & 2 & 1531 & CGA & $\mathrm{T}$ \\
\hline $\operatorname{trnL2}$ & F & 2978-3046 & 0 & 69 & & & $2974-3042$ & 0 & 69 & & & 2971-3039 & 0 & 69 & & \\
\hline $\operatorname{trnK}$ & $\mathrm{F}$ & $3731-3801$ & 0 & 71 & & & $3727-3797$ & 0 & 71 & & & $3724-3794$ & 0 & 71 & & \\
\hline $\operatorname{trnD}$ & $\mathrm{F}$ & $3801-3866$ & -1 & 66 & & & $3797-3861$ & -1 & 65 & & & $3794-3858$ & -1 & 65 & & \\
\hline atp 8 & F & $3867-4031$ & 0 & 165 & ATA & TAA & $3862-4023$ & 0 & 162 & ATA & TAA & $3859-4020$ & 0 & 162 & ATA & TAA \\
\hline atp6 & $\mathrm{F}$ & $4028-4702$ & -4 & 675 & ATA & TAA & $4020-4694$ & -4 & 675 & ATA & TAA & $4017-4691$ & -4 & 675 & ATA & TAA \\
\hline $\operatorname{cox} 3$ & F & $4705-5490$ & 2 & 786 & ATA & TAA & $4697-5482$ & 2 & 786 & ATA & TAA & $4694-5479$ & 2 & 786 & ATA & TAA \\
\hline $\operatorname{trn} G$ & $\mathrm{~F}$ & 5493-5558 & 2 & 66 & & & $5485-5550$ & 2 & 66 & & & $5482-5547$ & 2 & 66 & & \\
\hline nad3 & $\mathrm{F}$ & 5559-5912 & 0 & 354 & ATT & TAG & $5551-5904$ & 0 & 354 & ATT & TAG & $5548-5901$ & 0 & 354 & ATT & TAG \\
\hline $\operatorname{trn} A$ & $\mathrm{~F}$ & $5911-5978$ & -2 & 68 & & & $5903-5971$ & -2 & 69 & & & $5900-5968$ & -2 & 69 & & \\
\hline $\operatorname{trn} R$ & F & $5982-6047$ & 3 & 66 & & & $5975-6040$ & 3 & 66 & & & $5972-6037$ & 3 & 66 & & \\
\hline $\operatorname{trnN}$ & F & $6055-6120$ & 7 & 66 & & & $6045-6110$ & 4 & 66 & & & $6042-6107$ & 4 & 66 & & \\
\hline $\operatorname{trn} F$ & $\mathrm{R}$ & $6258-6324$ & 10 & 67 & & & $6238-6304$ & 2 & 67 & & & $6235-6301$ & 2 & 67 & & \\
\hline nad5 & $\mathrm{R}$ & $6325-8020$ & 0 & 1696 & ATA & $\mathrm{T}$ & 6305-7997 & 0 & 1693 & ATA & $\mathrm{T}$ & $6302-7994$ & 0 & 1693 & ATA & $\mathrm{T}$ \\
\hline $\operatorname{trnH}$ & $\mathrm{R}$ & $8063-8129$ & 42 & 67 & & & 8043-8109 & 45 & 67 & & & $8040-8106$ & 45 & 67 & & \\
\hline nad4 & $\mathrm{R}$ & $8131-9471$ & 1 & 1341 & ATG & TAA & $8111-9451$ & 1 & 1341 & ATG & TAA & 8108-9448 & 1 & 1341 & ATG & TAA \\
\hline nad4L & $\mathrm{R}$ & $9471-9746$ & -1 & 276 & ATA & TAA & $9451-9726$ & -1 & 276 & ATA & TAA & $9448-9723$ & -1 & 276 & ATA & TAA \\
\hline $\operatorname{trnT}$ & $\mathrm{F}$ & $9767-9832$ & 20 & 66 & & & $9747-9812$ & 20 & 66 & & & 9744-9809 & 20 & 66 & & \\
\hline $\operatorname{trn} P$ & $\mathrm{R}$ & 9833-9897 & 0 & 65 & & & 9813-9876 & 0 & 64 & & & 9810-9873 & 0 & 64 & & \\
\hline nad6 & $\mathrm{F}$ & $9900-10,424$ & 2 & 525 & ATA & TAA & $9879-10,403$ & 2 & 525 & ATA & TAA & $9876-10,400$ & 2 & 525 & ATA & TAA \\
\hline$c y t b$ & $\mathrm{~F}$ & $10,424-11,569$ & -1 & 1146 & ATG & TAA & $10,403-11,548$ & -1 & 1146 & ATG & TAA & $10,400-11,545$ & -1 & 1146 & ATG & TAA \\
\hline trnS2 & F & $11,575-11,645$ & 5 & 71 & & & $11,557-11,629$ & 8 & 73 & & & $11,554-11,626$ & 8 & 73 & & \\
\hline nad1 & $\mathrm{R}$ & $11,661-12,572$ & 15 & 912 & ATA & TAA & $11,645-12,556$ & 15 & 912 & ATA & TAA & $11,642-12,553$ & 15 & 912 & ATA & TAA \\
\hline $\operatorname{trnL1}$ & $\mathrm{R}$ & $12,594-12,662$ & 21 & 69 & & & $12,578-12,648$ & 21 & 71 & & & $12,575-12,645$ & 21 & 71 & & \\
\hline$r r n L$ & $\mathrm{R}$ & $12,638-13,992$ & -25 & 1355 & & & $12,649-13,984$ & 0 & 1336 & & & $12,646-13,981$ & 0 & 1336 & & \\
\hline $\operatorname{trn} V$ & $\mathrm{R}$ & $13,993-14,057$ & 0 & 65 & & & $13,984-14,048$ & -1 & 65 & & & $13,982-14,046$ & -1 & 65 & & \\
\hline$r r n S$ & $\mathrm{R}$ & $14,059-14,835$ & 1 & 777 & & & $14,048-14,825$ & -1 & 778 & & & $14,046-14,823$ & -1 & 778 & & \\
\hline $\begin{array}{c}A+ \\
\text { T-rich }\end{array}$ & & $14,836-15,389$ & & 554 & & & $14,826-15,612$ & & 787 & & & $14,824-15,496$ & & 673 & & \\
\hline
\end{tabular}

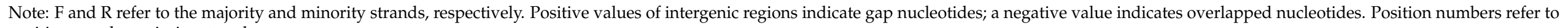
positions on the majority strand. 


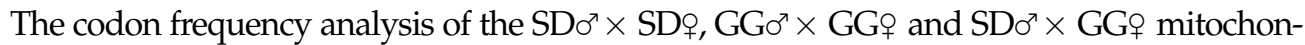
drial genomes showed that a total of 61 codons were used for transcription, with the absence of UAG (Figure 3). The GGo $\times$ GGq mitochondrial genomes had 10 more codons than SDo $\times$ $\mathrm{SD}$. However, the most frequently used codon in the three mitochondrial genomes was UUA for Leu, followed by AUU for Ile (Figure 3).

The fraction of codons encoding the hydrophobic amino acids (Met, Trp, Phe, Val, Leu,

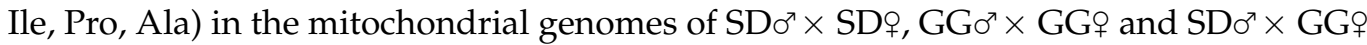
were $56.91 \%, 56.60 \%$ and $56.62 \%$, respectively (Figure 3 ), reflecting the biased usage of $\mathrm{A} / \mathrm{T}$ nucleotides and the hydrophobic nature of respiratory membrane complexes. The codon distribution patterns of the three compared mitochondrial genomes (Figure 3) were consistent with the finding that Ile, Leu, Phe, Ser, Asn and Tyr are the six amino acids most frequently used, whereas Lys is rare in Hepialidae [51].

All PCGs (except cox1) in the three mitochondrial genomes began with a canonical start codon (ATN or NTG). More specifically, eight PCGs (cox3, atp6, atp8, nad1, nad2, nad4L, nad5 and nad6) started with ATA, one PCG (nad3) with ATT, three PCGs (nad4, cytb and cox2) with ATG and one PCG (cox1) with CGA (Table 2). For the stop codon, nine PCGs (atp6, atp8, nad1, nad2, nad4, nad4L, nad6, cox3 and cob) were terminated with the typical stop codon TAA, while three PCGs (nad5, cox1 and cox2) located upstream of tRNAs ended with T, and one PCG (nad3) ended with TAG (Table 2).

Transfer RNA and ribosomal RNA genes. The predicted cloverleaf structures for 22 tRNA genes are presented in Figure S3. For three mitochondrial genomes of SD $\sigma^{7} \times \mathrm{SD}^{\circ}$, $\mathrm{GG}^{\top} \times$ GGo and SDo ${ }^{\top} \times$ GGq, the $r r n L(16 \mathrm{~S}$ rRNA) and $r r n S$ (12S rRNA) genes were identified, being 1355 bp, $1336 \mathrm{bp}$ and $1336 \mathrm{bp}$ and $777 \mathrm{bp}, 778 \mathrm{bp}$ and $778 \mathrm{bp}$ in size, respectively, falling into the reported range for the Hepialidae (1324-1375 bp, 740-781 bp) [41] (Table 2). The rrnL gene was located between trnL1 (TAG) and trnV (TAC), while rrnS was located between $\operatorname{trn} V$ (TAC) and the A + T-rich region (Table 2 and Figure 2). The $\mathrm{A}+\mathrm{T}$ percentages of rRNA in three mitochondrial genomes were $85.10 \%$ to $85.60 \%$. These rRNA characteristics are consistent with those observed in other Lepidoptera [41]. Twenty-two tRNAs were encoded in two mitochondrial genomes of the GGo $\times$ GGo and $\mathrm{SD}^{\top} \times$ GGq populations, ranging from $60 \mathrm{bp}$ to $73 \mathrm{bp}$ in size and spread across the entire genome. SDo $\times$ SD 9 ranged from $61 \mathrm{bp}$ to $71 \mathrm{bp}$. All tRNAs were shown to be folded into the expected clover-leaf secondary structure except for trnS1 (UCU), which lacks the dihydrouridine (DHU) loop (Figure S3). This feature is common to most of the available lepidopteran mitochondrial genomes [52].

Non-coding and overlapping genes. The complete mitochondrial genomes of

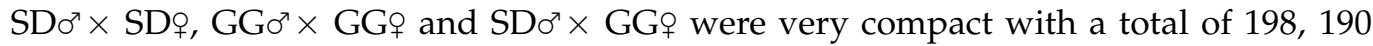
and 188 non-coding bp dispersed among 20, 17 and 17 pairs of neighboring genes ranging from 1 to $42 \mathrm{bp}, 1$ to $45 \mathrm{bp}$ and 1 to $45 \mathrm{bp}$, respectively (Table 2).

The longest spacer sequence was located between nad5 and trnH. A 15 bp intergenic spacer located between the trnS2 and nad1 contained the "ATACTAA" motif, which is a common feature across lepidopteran insects [53,54], but in Hepialidae species, the noncoding region contained an "ATACTA" sequence followed by T or C (Figure 4). The results are consistent with the report from [40]. In addition, the complete mitochondrial genomes

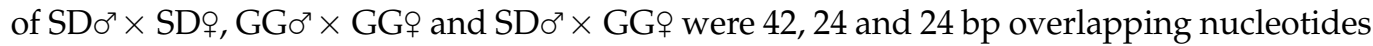
located in 7, 10 and 10 pairs of neighboring genes with a length from 1 to $25 \mathrm{bp}$. The longest overlapping nucleotides ( $25 \mathrm{bp}$ ) existed between $\operatorname{trnL1}$ and $r r n L$. The atp 8 and atp 6 had $4 \mathrm{bp}$ overlapping nucleotides (Figure S4). Those seven nucleotides "ATGATAA" are a common feature across the lepidopteran mitochondrial genomes [50]. 


\section{SD 1 x SD우 ATTTTATACTATATATTAATTATAA 15 \\ GG소 $\mathrm{x}$ GG우 ATTTTATACTACTTTATAATTATTA 15 \\ SD소 $\mathrm{xGG}$ 우 ATTTTATACTACTTTATAATTAATA 15}

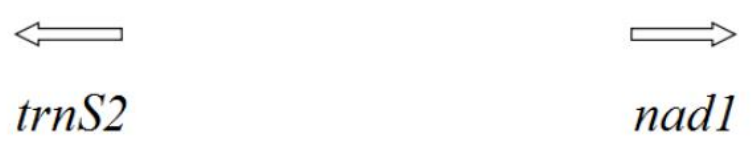

Figure 4. The highly conserved intergenic spacer located between the trnS2 and nad1.

$\mathrm{A}+\mathrm{T}$-rich region. The length and $\mathrm{A}+\mathrm{T}$ content of the $\mathrm{A}+\mathrm{T}$-rich regions were $554 \mathrm{bp}$ and $91.70 \%$ in $\mathrm{SD}{ }^{7} \times \mathrm{SD}$ \%, $787 \mathrm{bp}$ and $89.83 \%$ in GGo $\times$ GGo and $673 \mathrm{bp}$ and $90.64 \%$ in SDo $\times$ GG\% (Table 2). The A + T-rich region was located between the $r r n S$ and $\operatorname{trnI}$ genes. These repeat sequences accounted for some of the variations in $\mathrm{mt}$ genome length (Table 2). The A + T-rich region of T. renzhiensis is the longest of all the sequenced Lepidoptera mitochondrial genomes; the shortest is $319 \mathrm{bp}$ in O. lunifer [55]. This variation in length could be related to the number and lengths of tandem repeats in the control region [56]. SDor $\times \mathrm{SD}$ ? had no repeating sequences (Table 2). Two repeated sequences $(114 \mathrm{bp})$ in GGo $\times$ GGo were detected, each with one more base A. Compared with $\mathrm{GGo}^{\top} \times \mathrm{GG}$ 우, $\mathrm{SD} 0^{7} \times \mathrm{GG}$ ㅇ lacked a set of repeated sequences, and two repeated sequences were $113 \mathrm{bp}$ and $114 \mathrm{bp}$, respectively, which were only one A base apart (Table 2). This sequence repetition is not unique. There are four repetitive sequences of $118 \mathrm{bp}$ in length in T. sejilaensis, four $107 \mathrm{bp}$ repeat sequences in A. yunnanensis, eight $113 \mathrm{bp}$ repeats in T. renzhiensis [36], six 112 bp repeats in T. gonggaensis [39] and five 119 bp repeats in T. pui [37].

\subsection{Phylogenetic Relationships and Taxonomic Relation}

To confirm the evolutionary position of the host insects of $O$. sinensis, a phylogenetic tree of 11 species using published mitochondrial genomes (10 Hepialidae, 1 outgroup) and three genomes from the present study was constructed based on the concatenated nucleotides' alignment of 13 PCGs or each PCG by the ML method. SDor $\times$ SD 9 was classified into a separate clade and $\mathrm{GG}^{-} \times \mathrm{GG}$ ㅇ and $\mathrm{SD} 0^{-} \times \mathrm{GG}$ ㅇ into another separate clade (Figure 5 and Figure S5). Thus, $\mathrm{SD} 0^{7} \times \mathrm{SD}$ ㅇ and GGơ $\times$ GGㅇ were confirmed to be different species. SDo ${ }^{r} \times$ SDo was considered to be an undescribed Thitarodes species according to the present database, and GGor $\times$ GGo was identified as T. shambalenensis by the cox1 fragment. The genetical

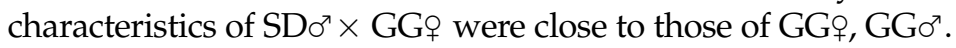

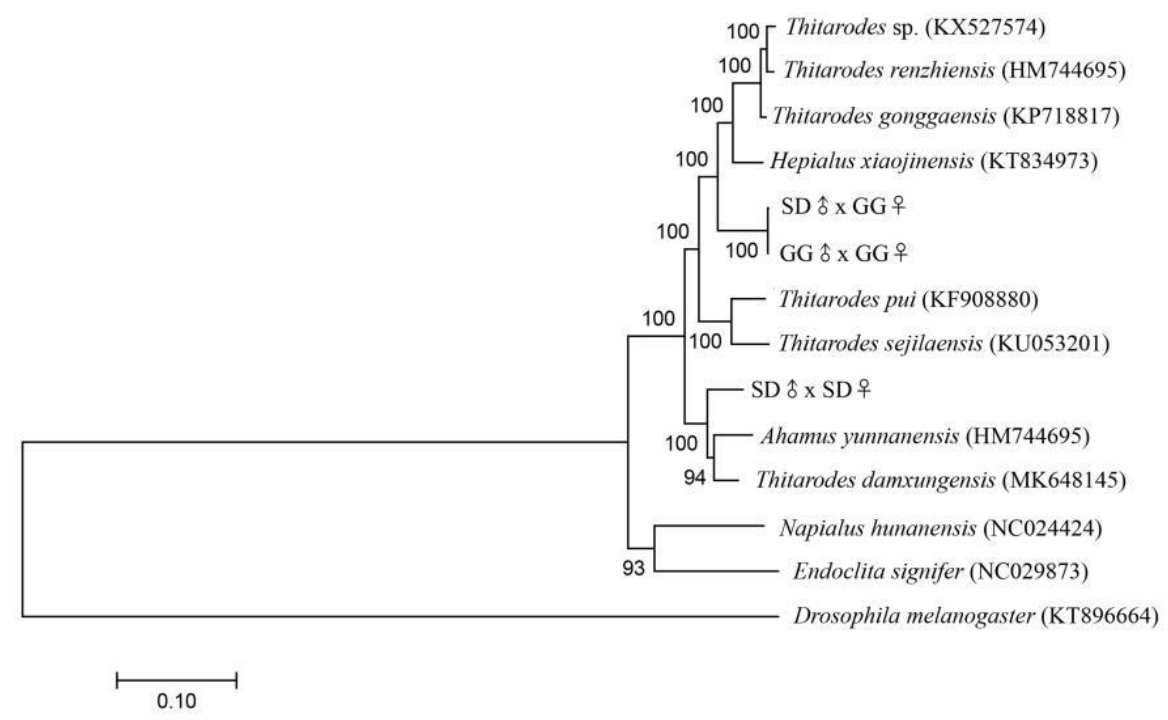

Figure 5. The phylogenetic relationship constructed by the amino acid sequence derived from 13 PCGs among 13 mitogenomes of Hepialidae insects and 1 outgroup. 


\section{Discussion}

T. shambalaensis (GGor $\times$ GGo) identified by morphology (male genitalia) and genetic cox1 phylogeny [43] and an undescribed Thitarodes species (SDo $\times$ SD우) live in different locations in the Tibetan Plateau. In the present study, interspecific hybridization between these two distinct ghost moth species was demonstrated in the laboratory. The developmental performance of the studied Thitarodes populations was influenced by the hybridization, whereas the larval sensitivity to the fungal infection of the inbred populations was affected by the parental populations.

Hybridization between two related insect species is common in the laboratory or in the field; for instance, interspecific hybridization has been reported between Helicoverpa armigera and Helicoverpa assulta [57], Nasutitermes corniger and Nasutitermes ephratae [58], Coptotermes formosanus and Coptotermes gestroi [59] and between Reticulitermes flaviceps and Reticulitermes chinensis [43]. The red imported fire ant Solenopsis invicta, black imported fire ant Solenopsis richteri and their hybrid (S. invicta $\times$ S. richteri) are present in the field in Tennessee, USA [60]. The studied T. shambalaensis and Thitarodes sp. do not share a habitat in the Tibetan Plateau. It is speculated that reproductive individuals of the two ghost moth species may not have the chance to hybridize in nature because of the limited flying capacity of the adults. Surprisingly, these two species could mate, and the resulting hybrids produced a next generation. Whereas the inbred $\mathrm{SD} \sigma^{7} \times \mathrm{SD}$ laboratory population was very weak $(\mathrm{I}=0.01)$, the $\mathrm{SD} \sigma^{7} \times \mathrm{GG}$ o population exhibited a higher population trend index $(I=0.32)$, implying growth potential to a certain extent from $\mathrm{GG}^{7} \times \mathrm{GG}$, whose inbred population had a higher population trend index $(\mathrm{I}=2.25)$. Hybridization may thus provide an effective method to create Thitarodes/Hepialus populations with increased growth potential for the improved artificial production of the insect hosts.

Why these distinct Thitarodes species can be hybridized in the laboratory remains unknown. Species are defined to be groups of interbreeding natural populations that are reproductively isolated from other such groups [61]. The mechanism of pre-zygotic or postzygotic reproductive isolation is considered to be involved in speciation [62]. Pre-zygotic reproductive isolation includes ecological and geographical habitat isolation, mating season or time difference, genitalia structure isolation, gamete isolation and mating or mating behavior isolation, whereas post-zygotic reproductive isolation includes survival limitation, infertility and depression of the hybrids [62]. Certainly, in this study, the hybridization of two Thitarodes species occurred in the laboratory, not in nature. The resulting hybrids also produced a next generation, indicating that the post-zygotic reproductive isolation may not prevent hybridization between two Thitarodes species, even in nature. Thus, the successful hybridization of these two species should depend on overcoming the pre-zygotic reproductive isolation, especially geographical habitat isolation and mating behavior isolation. Although reproductive isolation can evolve in a number of different ways, speciesspecific mate recognition by sex pheromones is believed to be a key element [63]. Similar recognition systems are a prerequisite for the interspecific interactions of closely related species in nature. However, in the laboratory, heterospecific partners can compulsively interact without the species-specific mate recognition. It appears that these two Thitarodes species can overcome the different genitalia structure and gamete isolation in the prezygotic phase and reproductive isolation in the post-zygotic phase in the laboratory. These results demonstrate the complexity of reproductive isolation and provide useful cues for further study in the speciation mechanism.

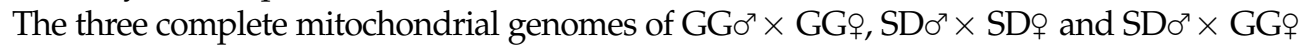
differ not only in the size of the genome but also in the A + T-rich region with repeat sequences. So far, eight Thitarodes / Hepialus mitochondrial genomes are sequenced, including T. renzhiensis, T. yunnanensis, T. pui, H. xiaojinensis, H. gonggaensis, T. sejilaensis, an undescribed Thitarodes sp. and T. damxungensis [21]. Based on the phylogenetic tree constructed by 13 PCGs from the previously described genomes, $\mathrm{GG}^{\top} \times \mathrm{GG}$ ㅇ was identified as T. shambalenensis, and $\mathrm{SD} 0^{7} \times \mathrm{SD}$ ㅇ was considered to be an undescribed Thitarodes species (Figure 5), given the reasonable threshold for inter-species variation (2.5\% genetic distance) $[43,64]$. Interestingly, $\mathrm{SD}{ }^{\top} \times \mathrm{GG}$ ㅇ was close to 
$\mathrm{GGo}^{\top} \times$ GG, according to the genetical similarities, which confirms the maternal inheritance of mitochondrial DNA. The sizes of eleven Thitarodes/Hepialus mitochondrial genomes, including the three genomes in the present study, are variable from 15,290 bp in T. sejilaensis [40] to 16,280 bp in Thitarodes sp. [41]. Likewise, reports that the mitochondrial genomes of the hybrids of bream fishes [65] or Acipenser schrenckii () $\times$ Huso dauricus [66] are variable in gene sizes. Why the Thitarodes hybrid and the populations sharing the same mother have different mtDNAs in genome length, $\mathrm{A}+\mathrm{T}$ content and the sizes of the $\mathrm{A}+\mathrm{T}$-rich region needs further study.

The sizes of the $\mathrm{A}+\mathrm{T}$-rich region are $787 \mathrm{bp}$ with two repetitive sequences of 114 $\mathrm{bp}, 554 \mathrm{bp}$ without repetitive sequences and $673 \mathrm{bp}$ without repetitive sequences in $\mathrm{GG}^{\top} \times \mathrm{GG}+\mathrm{SD}^{\top} \times \mathrm{SD}+$ and $\mathrm{SD}^{\top} \times \mathrm{GG}$, respectively. The $\mathrm{A}+\mathrm{T}$ region of $\mathrm{SD}^{\top} \times$ SDo contains non-repetitive sequences only, just like that in T. damxungensis [21]. It seems that the sizes of the $\mathrm{A}+\mathrm{T}$-rich region with repetitive sequences are quite different among the available Hepialidae mitogenomes, e.g., those in T. damxungensis ( $545 \mathrm{bp}$ without repetitive sequences), T. pui (1030 bp with five $119 \mathrm{bp}$ repeat units), T. sejilaensis ( $484 \mathrm{bp}$ with four $118 \mathrm{bp}$ repeat units), T. yunnanensis (1000 bp with four $107 \mathrm{bp}$ repeat units), T. xiaojinensis (634 bp with four 118 bp repeat units), T. gonggaensis (1133 bp with six 112 bp repeat units), T. renzhiensis (1358 bp with eight $113 \mathrm{bp}$ repeat units) and an undescribed Thitarodes. sp. (1472 bp with nine 112 bp repeat units) [21]. The relatively fast evolutionary rate in this A + $\mathrm{T}$ region appears to cause significant size variation [41].

\section{Conclusions}

In conclusion, the present study demonstrated that interspecific hybridization occurred under laboratory conditions between two allopatric and morphologically distinct ghost moth species T. shambalaensis and Thitarodes sp. Secondly, we found that the offspring produced by hybridization may display increased growth potential at least from one of the parent populations, which would greatly improve the cultivation of Thitarodes insects for the artificial production of Chinese cordyceps. Finally, our study demonstrated that the mitochondrial genome from the hybrid is different from those of its parents in several features (genome length, $\mathrm{A}+\mathrm{T}$ content and the sizes of the $\mathrm{A}+\mathrm{T}$-rich region) and maternal inheritance.

Supplementary Materials: The following are available online at https:/ / www.mdpi.com/article/10 .3390 /insects12111046/s1, Figure S1: Comparison of the fresh weights of the larvae of inbred and hybrid populations during the culture times, Figure S2: Comparison of the larval survival rates of the larvae of inbred and hybrid populations, Figure S3: Secondary structures of 22 tRNAs encoded by the Thitarodes mitochondrial genomes, Figure S4: Alignment of overlapping region between atp 8 and atp 6 across three Thitarodes populations, Figure S5: The phylogenetic relationship constructed by the amino acid sequence derived from each PCG among 13 mitogenomes of Hepialidae insects and 1 outgroup, Table S1: Egg characters from the inbred and hybrid populations, Table S2: Mummification rates of the inbred larvae at 120 days post infection with O. sinensis fungus. Table S3: Fresh weight of infected and uninfected larvae at 120 days post infection with different $O$. sinensis fungal strains.

Author Contributions: R.H. and P.D.C. designed and coordinated the research. H.W. and L.C. collected the samples. H.W. conducted the research. H.W. and M.H. analyzed the data. R.H., P.D.C. and H.W. wrote the manuscript. All authors have read and agreed to the published version of the manuscript.

Funding: This work was supported by the GDAS Special Project of Science and Technology Development (2019GDASYL-0103056 and 2020GDASYL20200103097), Research and Development Projects in Key Fields in Guangdong Province (2020B1111580001), and Student Project of the Institute of Zoology, Guangdong Academy of Sciences (GIABR-pyjj201810).

Institutional Review Board Statement: Not applicable.

Informed Consent Statement: Not applicable.

Data Availability Statement: Exclude this statement. 
Acknowledgments: The authors gratefully acknowledge Zhiwen Zou from Nanchang University for the assistance in preparing the photomicrographs (Figure 1) and for useful discussions.

Conflicts of Interest: The authors declare no conflict of interest.

\section{References}

1. Paterson, R.R.M. Cordyceps-A traditional Chinese medicine and another fungal therapeutic biofactory? Phytochemisty 2008, 69, 1469-1495. [CrossRef]

2. Dong, C.H.; Yao, Y.J. On the reliability of fungal materials used in studies on Ophiocordyceps sinensis. J. Ind. Microbiol. Biotechnol. 2011, 38, 1027-1035. [CrossRef]

3. Shrestha, U.B.; Bawa, K.S. Impact of climate change on potential distribution of Chinese Caterpillar fungus (Ophiocordyceps sinensis) in Nepal Himalaya. PLoS ONE 2014, 9, e106405.

4. RiChou, H.; Hua, W.; HaiPing, T.; XueHong, Q.; GuiQing, L.; ZhongChen, R.; Li, C. Research on Chinese cordyceps during the past 70 years in China. Chin. J. Appl. Entomol. 2019, 56, 849-883.

5. $\quad$ Liang, H.H.; Cheng, Z.; Yang, X.L.; Li, S.; Ding, Z.Q.; Zhou, T.S.; Zhang, W.J.; Chen, J.K. Genetic diversity and structure of Cordyceps sinensis populations from extensive geographical regions in China as revealed by inter-simple sequence repeat markers. J. Microbiol. 2008, 5, 549-556. [CrossRef] [PubMed]

6. Winkler, D. Caterpillar fungus (Ophiocordyceps sinensis) production and sustainability on the Tibetan Plateau and in the Himalayas. Asian Med. 2009, 5, 291-316. [CrossRef]

7. Holliday, J.; Cleaver, M. Medicinal value of the caterpillar fungi species of the genus Cordyceps (Ascomycetes): A review. Int. J. Med. Mushrooms 2008, 10, 219-234. [CrossRef]

8. Li, W.J.; Dong, C.H.; Liu, X.Z.; Li, Q.P.; Xia, J.M.; Liang, L. Research advances in artificial cultivation of Chinese cordyceps. Mycosystema 2016, 35, 375-387.

9. Baral, B. Entomopathogenicity and biological attributes of Himalayan treasured fungus Ophiocordyceps sinensis (Yarsagumba). J. Fungi 2017, 3, 4. [CrossRef]

10. Pouliota, M.; Pyakurela, D.; Smith-Halla, C. High altitude organic gold: The production network for Ophiocordyceps sinensis from far-western Nepal. J. Ethnopharmacol. 2018, 218, 59-68. [CrossRef]

11. Qin, Q.L.; Zhou, G.L.; Zhang, H.; Meng, Q.; Zhang, J.H.; Wang, H.T.; Miao, L.; Li, X. Obstacles and approaches in artificial cultivation of Chinese cordyceps. Mycology 2018, 1, 7-9. [CrossRef]

12. Chen, L.T.; Tang, J.J.; Mao, J.L. A preliminary study on the biology of the "insect herb", Hepialus armoricanus Oberthür. Acta Entomol. Sin. 1973, 6, 198-202.

13. Quan, Q.M.; Chen, L.L.; Wang, X.; Li, S.; Yang, X.L.; Zhu, Y.G.; Wang, M.; Cheng, Z. Genetic diversity and distribution patterns of host insects of Caterpillar fungus Ophiocordyceps sinensis in the Qinghai-Tibet plateau. PLOS ONE 2014, 9, e92293.

14. Zhang, Y.J.; Zhang, S.; Li, Y.L.; Ma, S.L.; Wang, C.S.; Xiang, M.C.; Liu, X.; An, Z.Q.; Xu, J.P.; Liu, X.Z. Phylogeography and evolution of a fungal-insect association on the Tibetan Plateau. Mol. Ecol. 2014, 23, 5337-5355. [CrossRef]

15. Quan, Q.M.; Wang, Q.X.; Zhou, X.L.; Li, S.; Yang, X.L.; Zhu, Y.G.; Cheng, Z. Comparative phylogenetic relationships and genetic structure of the Caterpillar fungus Ophiocordyceps sinensis and its host insects inferred from multiple gene sequences. J. Microbiol. 2014, 52, 99-105. [CrossRef]

16. Yang, D.R.; Li, C.D.; Shu, C.; Yang, Y.X. Studies on the Chinese species of the genus Hepialus and their geographical distribution. Acta Entomol. Sin. 1996, 39, 413-422.

17. Wang, X.L.; Yao, Y.J. Host insect species of Ophiocordyceps sinensis: A review. Zookeys 2011, 127, 43-59.

18. Li, J.F.; Zhang, G.R. Life table of the experimental population of Thitarodes pui (Lepidoptera, Hepialidae), a host species of Ophiocordyceps sinensis. J. Environ. Entomol. 2012, 34, 386-389.

19. Li, Q.P.; He, Y.; Liu, J.M.; Xia, J.M.; Li, W.J.; Liu, X.Z. Hybrid breeding of high quality of Hepialus sp., the host of Ophiocordyceps sinensis, and prevention of the host insect reproductive degradation. Mycosystema 2016, 35, 456-466.

20. Tao, Z.; Cao, L.; Zhang, Y.; Ye, Y.S.; Han, R.C. Laboratory rearing of Thitarodes armoricanus and Thitarodes jianchuanensis (Lepidoptera: Hepialidae), hosts of the Chinese medicinal fungus Ophiocordyceps sinensis (Hypocreales: Ophiocordycipitaceae). J. Econ. Entomol. 2016, 109, 176-181. [CrossRef]

21. Zhang, M.; Gao, Z.M.; Yin, J.; Zhang, T.M.; Zhang, X.Y.; Yuan, D.W.; Li, T.; Zhong, Y.; Ma, E.B.; Ren, Z.M. Complete mitochondrial genome of two Thitarodes species (Lepidoptera, Hepialidae), the host moths of Ophiocordyceps sinensis and phylogenetic implications. Int. J. Biol. Macromol. 2019, 140, 794-807. [CrossRef] [PubMed]

22. Huang, T.F.; Fu, S.Q.; Luo, Q.M. Experiments on the feeding habits of Hepialus armoricanus larva in Kangding. Sichuan J. Zool. 1989, 8, 8-10.

23. Yue, K.; Ye, M.; Lin, X.; Zhou, Z.J. The artificial cultivation of medicinal caterpillar fungus, Ophiocordyceps sinensis (Ascomycetes): A review. Int. J. Med. Mushrooms 2013, 15, 425-434. [CrossRef] [PubMed]

24. Zhou, Y.J.; Xiang, L.; Zhou, Z.J.; Dai, Y.; Han, K.H.; Zhu, T.H. Biological characteristics of four generation artificial swift moth adults. J. Northeast For. Univ. 2014, 42, 112-115.

25. Cao, L.; Han, R.C. A Method for Artificial Cultivation of Fruiting Bodies of Ophiocordyceps sinensis. Chinese Patent ZL201410289703.0.2014-06-25, 20 May 2015. 
26. Liu, G.Q.; Han, R.C.; Cao, L. Artificial cultivation of the Chinese Cordyceps from injected ghost moth larvae. Environ. Entomol. 2019, 5, 1088-1094. [CrossRef] [PubMed]

27. Liu, G.Q.; Cao, L.; Qiu, X.H.; Han, R.C. Quorum sensing activity and hyphal growth by external stimuli in the entomopathogenic fungus Ophiocordyceps sinensis. Insects 2020, 11, 205. [CrossRef]

28. Ruiz-Herrera, J.; Pérez-Rodríguez, F.; Velez-Haro, J. The signaling mechanisms involved in the dimorphic phenomenon of the Basidiomycota fungus Ustilago maydis. Int. Microbiol. 2020, 23, 123-126. [CrossRef]

29. Boucias, D.; Liu, S.; Meagher, R.; Baniszewski, J. Fungal dimorphism in the entomopathogenic fungus Metarhizium rileyi: Detection of an in vivo quorum-sensing system. J. Invertebr. Pathol. 2016, 136, 100-108. [CrossRef]

30. Meng, Q.; Yu, H.Y.; Zhang, H.; Zhu, W.; Wang, M.L.; Zhang, J.H.; Zhou, G.L.; Li, X.; Qin, Q.L.; Hu, S.N.; et al. Transcriptomic insight into the immune defenses in the ghost moth, Hepialus xiaojinensis, during an Ophiocordyceps sinensis fungal infection. Insect Biochem. Mol. Biol. 2015, 64, 1-15. [CrossRef]

31. Rao, Z.C.; Cao, L.; Qiu, X.H.; Han, R.C. Comparative transcriptome analysis reveals molecular strategies of ghost moth Thitarodes armoricanus in response to hypoxia and anoxia. J. Insect Physiol. 2019, 112, 23-34. [CrossRef]

32. Krzywinski, J.; Grushko, O.G.; Besansky, N.J. Analysis of the complete mitochondrial DNA from Anopheles funestus: An improved dipteran mitochondrial genome annotation and a temporal dimension of mosquito evolution. Mol. Phylogenet. Evol. 2006, 39, 417-423. [CrossRef]

33. Wolstenholme, D.R. Genetic novelties in mitochondrial genomes of multicellular animals. Curr. Opin. Genet. Dev. 1992, 2, 918-925. [CrossRef]

34. Boore, J.L. Animal mitochondrial genomes. Nucleic Acids Res. 1999, 27, 1767-1780. [CrossRef] [PubMed]

35. Sheffield, N.C.; Song, H.; Cameron, S.L.; Whiting, M.F. A Comparative analysis of mitochondrial genomes in Coleoptera (Arthropoda: Insecta) and genome descriptions of six new beetles. Mol. Biol. Evol. 2008, 25, 2499-2509. [CrossRef] [PubMed]

36. Cao, Y.Q.; Ma, C.; Chen, J.Y.; Yang, D.R. The complete mitochondrial genomes of two ghost moths, Thitarodes renzhiensis and Thitarodes yunnanensis: The ancestral gene arrangement in Lepidoptera. BMC Genom. 2012, 13, 276. [CrossRef] [PubMed]

37. Yi, J.Q.; Que, S.Q.; Xin, T.R.; Xia, B.; Zou, Z.W. Complete mitochondrial genome of Thitarodes pui (Lepidoptera: Hepialidae). Mitochondrial DNA 2016, 27, 109-110. [CrossRef]

38. Chen, S.J.; Shi, P.; Zhang, D.L.; Lu, Z.H.; Li, L.Y. Complete mitochondrial genome of Hepialus xiaojinensis (Lepidoptera: Hepialidae). Mitochondrial DNA 2017, 28, 305-306. [CrossRef] [PubMed]

39. Shi, P.; Lu, Z.H.; He, Y.C.; Chen, S.J.; Qin, S.R.; Qing, Y.L.; Yan, J. Complete mitochondrial genome of Hepialus gonggaensis (Lepidoptera: Hepialidae), the host insect of Ophiocordyceps sinensis. Mitochondrial DNA 2016, 27, 4205-4206. [CrossRef] [PubMed]

40. Zou, Z.W.; Min, Q.; Cheng, S.Y.; Xin, T.R.; Xia, B. The complete mitochondrial genome of Thitarodes sejilaensis (Lepidoptera: Hepialidae), a host insect of Ophiocordyceps sinensis and its implication in taxonomic revision of Hepialus adopted in China. Gene 2016, 601, 44-55. [CrossRef]

41. Kang, X.C.; Hu, Y.Q.; Hu, J.; Hu, L.Q.; Wang, F.; Liu, D.B. The mitochondrial genome of the lepidopteran host cadaver (Thitarodes sp.) of Ophiocordyceps sinensis and related phylogenetic analysis. Gene 2017, 598, 32-42. [CrossRef]

42. Zou, Z.W.; Liu, X.; Zhang, G.R. Revision of taxonomic system of Hepialus (Lepidoptera, Hepialidae) currently adopted in China. J. Hunan Univ. Sci. Technol. 2010, 25, 114-120.

43. Wang, Z.Y.; Zhuang, H.L.; Wang, M.; Pierce, N.E. Thitarodes shambalaensis sp. nov. (Lepidoptera, Hepialidae): A new host of the caterpillar fungus Ophiocordyceps sinensis supported by genome-wide SNP data. Zookeys 2019, 885, 89-113. [CrossRef] [PubMed]

44. Wu, H.; Rao, Z.C.; Cao, L.; De Clercq, P.; Han, R.C. Infection of Ophiocordyceps sinensis fungus causes dramatic changes in the microbiota of its Thitarodes Host. Fornt. Microbiol. 2020, 11, 577268. [CrossRef]

45. Simon, C.; Frati, F.; Beckenbach, A.; Crespi, B.; Liu, H.; Flook, P. Evolution, weighting, and phylogenetic utility of mitochondrial gene sequences and a compilation of conserved polymerase chain reaction primers. Ann. Entomol. Soc. Am. 1994, 87, 651-701. [CrossRef]

46. Folmer, O.; Black, M.; Hoeh, W.; Lutz, R.; Vrijenhoek, R. DNA primers for amplification of mitochondrial cytochrome c oxidase subunit I from diverse metazoan invertebrates. Mol. Mar. Biol. Biotechnol. 1994, 3, 294-299. [PubMed]

47. Lunau, S.; Stoessel, S.; Schmidt-Peisker, A.J.; Ehlers, R.-U. Establishment of monoxenic inocula for scaling up in vitro cultures of the entomopathogenic Nematodes Steinernema spp. and Heterorhabditis spp. Nematologica 1993, 39, 385-399. [CrossRef]

48. Cao, L.; Ye, Y.S.; Han, R.C. Fruiting body production of the medicinal fungus Ophiocordyceps sinensis in artificial medium. Int. J. Med. Mushrooms 2015, 17, 1107-1112. [CrossRef]

49. Lobry, J.R. Asymmetric substitution patterns in the two DNA strands of bacteria. Mol. Biol. Evol. 1996, 13, 660-665. [CrossRef]

50. Lu, H.F.; Su, T.J.; Luo, A.R.; Zhu, C.D.; Wu, C.S. Characterization of the complete mitochondrion genome of diurnal moth Amata emma (Butler) (Lepidoptera: Erebidae) and its phylogenetic implications. PLoS ONE 2013, 8, e72410. [CrossRef]

51. Dai, L.S.; Qian, C.; Zhang, C.F.; Wang, L.; Wei, G.Q.; Li, J.; Zhu, B.J.; Liu, C.L. Characterization of the complete mitochondrial genome of Cerura menciana and comparison with other lepidopteran insects. PLoS ONE 2015, 10, e0132951.

52. Cong, Q.; Grishin, N.V. The complete mitochondrial genome of Lerema accius and its phylogenetic implications. PeerJ 2016, 4, e1546. [CrossRef]

53. Cameron, S.L.; Whiting, M.F. The complete mitochondrial genome of the tobacco hornworm, Manduca sexta, (Insecta: Lepidoptera: Sphingidae), and an examination of mitochondrial gene variability within butterflies and moths. Gene 2008, 408, 112-123. [CrossRef] [PubMed] 
54. Liao, F.; Wang, L.; Wu, S.; Li, Y.P.; Zhao, L.; Huang, G.M.; Niu, C.J.; Liu, Y.Q.; Li, M.G. The complete mitochondrial genome of the fall webworm, Hyphantria cunea (Lepidoptera: Arctiidae). Int. J. Biol. Sci. 2010, 6, 172-186. [CrossRef] [PubMed]

55. Salvato, P.; Simonato, M.; Battisti, A.; Negrisolo, E. The complete mitochondrial genome of the bag-shelter moth Ochrogaster lunifer (Lepidoptera, Notodontidae). BMC Genom. 2008, 9, 331. [CrossRef] [PubMed]

56. Kotzé, A.; Grobler, J.P.; Dalton, D.L.; Labuschagne, C. The complete sequence of the mitochondrial genome of the African Penguin (Spheniscus demersus). Gene 2014, 534, 113-118.

57. Zhao, X.C.; Dong, J.F.; Tang, Q.B.; Yan, Y.H.; Gelibic, I.; Van Loon, J.J.A.; Wang, C.Z. Hybridization between Helicoverpa armigera and Helicoverpa assulta (Lepidoptera: Noctuidae): Development and morphological characterization of F1 hybrids. Bull. Entomol. Res. 2005, 95, 409-416. [CrossRef]

58. Hartke, T.R.; Rosengaus, R.B. Heterospecific pairing and hybridization between Nasutitermes corniger and N. ephratae. Naturwissenschaften 2011, 98, 745-753. [CrossRef]

59. Chouvence, T.; Helmick, E.E.; Su, N.Y. Hybridization of two major termite invaders as a consequence of human activity. PLoS ONE 2015, 10, e0120745.

60. Pandey, M.; Addesso, K.M.; Archer, R.S.; Valles, S.M.; Baysal-Gurel, F.; Ganter, P.F.; Youssef, N.N.; Oliver, J.B. Worker size, geographical distribution, and introgressive hybridization of invasive Solenopsis invicta and Solenopsis richteri (Hymenoptera: Formicidae) in Tennessee. Environ. Entomol. 2019, 48, 727-732. [CrossRef]

61. Ernst, M. The biological meaning of species. Biol. J. Linn. Soc. 1969, 1, 311-320.

62. Dobzhansky Theodosius, D. Genetic Organization. BioScience 1970, $20,777$.

63. Adams, S.A.; Tsutsui, N.D. The evolution of species recognition labels in insects. Philos. Trans. R. Soc. B 2020, $375,20190476$. [CrossRef] [PubMed]

64. Kartavtsev, Y.P. Sequence divergence at mitochondrial genes in animals: Applicability of DNA data in genetics of speciation and molecular phylogenetics. Mar. Genom. 2011, 4, 71-81. [CrossRef] [PubMed]

65. Zhang, W.Z.; Xiong, X.M.; Zhang, X.J.; Wan, S.M.; Guan, N.N.; Nie, C.H.; Zhao, B.W.; Hsiao, C.D.; Wang, W.M.; Gao, Z.X. Mitochondrial genome variation after hybridization and differences in the first and second generation hybrids of bream fishes. PLoS ONE 2016, 11, e0158915. [CrossRef] [PubMed]

66. Li, S.; Guo, W.T.; Liu, X.Q.; Qu, H.T.; Guan, M.; Su, W. Complete mitochondrial genome of the hybrid of Acipenser schrenckii (\$) $\times$ Huso dauricus ( $\left.\sigma^{7}\right)$. Mitochondrial DNA 2016, 27, 2887-2888. [CrossRef] [PubMed] 